\title{
MARTIN COMPACTIFICATIONS AND QUASICONFORMAL MAPPINGS
}

\author{
SHIGEO SEGAWA AND TOSHIMASA TADA
}

Dedicated to Professor Yukio Kusunoki on the occasion of his 60 th birthday.

\begin{abstract}
It is shown that there exists a quasiconformal mapping $T$ of a Riemann surface $R_{1}$ onto another $R_{2}$ such that $T$ cannot be extended to a homeomorphism of the Martin compactification $R_{1}^{*}$ of $R_{1}$ onto that $R_{2}^{*}$ of $R_{2}$.
\end{abstract}

It has long been asked whether a quasiconformal mapping between two Riemann surfaces can be extended to a homeomorphism between two Martin compactifications of the given surfaces. In literature this interesting problem was first explicitly stated in an expository paper by Royden [5]. Since then the problem seems to have been open and here we wish to settle the question in the negative. Namely,

THEOREM. There exists a quasiconformal mapping $T$ of a Riemann surface $R_{1}$ onto another $R_{2}$ such that $T$ cannot be extended to a homeomorphism of the Martin compactification $R_{1}^{*}$ of $R_{1}$ onto that $R_{2}^{*}$ of $R_{2}$.

In passing we remark that the Theorem is invalid if the Martin compactification is replaced by the Royden compactification (cf., e.g., Sario and Nakai [6]). Concerning the other compactifications such as Kuramochi and Wiener compactifications the question seems to be entirely open (cf., e.g., Constantinescu and Cornea [3]).

The proof of the Theorem is achieved by giving examples of $\left(R_{1}, R_{2}, T\right)$. Actually we can choose $R_{1}$ and $R_{2}$ to be plane regions of rather simple characters which were considered by Ancona [1]. In $\S 1$ an example of Ancona, mentioned above, is stated as the first Lemma. A minimality criterion also given by Ancona [2] is then stated as the second Lemma (in §2). Based upon these two preliminary results the construction of $\left(R_{1}, R_{2}, T\right)$ is carried over in $\S 3$.

1. We start by fixing notation. Let $\alpha$ and $\beta$ be positive numbers with $\alpha+\beta<\pi$. Using decreasing sequences $\left\{t_{n}\right\}_{1}^{\infty}$ and $\left\{t_{n}^{\prime}\right\}_{1}^{\infty}$ in $(0,1 / 2]$ with $\lim t_{n}=\lim t_{n}^{\prime}=0$, we consider the radial slits

$$
T_{n}=\left\{r e^{i \alpha} ; t_{2 n} \leqslant r \leqslant t_{2 n-1}\right\}, \quad T_{n}^{\prime}=\left\{r e^{-i \alpha} ; t_{2 n}^{\prime} \leqslant r \leqslant t_{2 n-1}^{\prime}\right\}
$$

Received by the editors February 2, 1984.

1980 Mathematics Subject Classification. Primary 30F25; Secondary 30C60.

Key words and phrases. Martin compactification, quasiconformal mapping. 
and the region

$$
\Omega_{0}=\left\{r e^{i \theta} ; 0<r<2,|\theta|<\alpha+\beta\right\}-\bigcup_{n=1}^{\infty}\left(T_{n} \cup T_{n}^{\prime}\right) .
$$

The following surprisingly interesting fact is due to Ancona [1].

LEMMA. If $2 \alpha<\beta$, then there exist sequences $\left\{t_{n}\right\},\left\{t_{n}^{\prime}\right\}$ in $(0,1 / 2]$ such that the Martin boundary of $\Omega_{0}$ lying over $z=0$ contains two distinct minimal points $\zeta$ and $\zeta^{\prime}$, and

$$
\lim _{n \rightarrow \infty} \frac{1}{2} t_{n}=\zeta, \quad \lim _{n \rightarrow \infty} \frac{1}{2} t_{n}^{\prime}=\zeta^{\prime}
$$

2. We need another result of Ancona [2] on a criterion of minimality. Let $\Omega$ be a plane region and $x$ a relative boundary point of $\Omega$. Then the above cited criterion is

LEMMA. If there exists an open disk $B(y, r)$ of center $y$ and radius $r$ with the property that $B(y, r) \subset \Omega$ and $x \in \partial B(y, r)$, the relative boundary of $B(y, r)$, then there exists a minimal Martin boundary point $\zeta$ lying over $x$ with

$$
\lim _{t \rightarrow 0}(x+t(y-x))=\zeta \text {. }
$$

3. For any $\alpha$ and $\beta$ with $0<2 \alpha<\beta$ and $\alpha+\beta<\pi$, consider a $C^{1}$ function $f(\theta)$ on $[-\beta-\alpha, \alpha+\beta]$ such that $f( \pm(\alpha+\beta))= \pm \pi, f( \pm \alpha)= \pm \pi / 2, f(0)=0$, and

$$
C^{-1} \leqslant f^{\prime}(\theta)=\frac{d}{d \theta} f(\theta) \leqslant C
$$

for a constant $C$ with $C>1$. Let $\Omega_{0}$ be a region for which the Lemma in $\S 1$ is valid, and let $\psi$ be the mapping from $\Omega_{0}$ to $\psi\left(\Omega_{0}\right)$ defined by

$$
\psi\left(r e^{i \theta}\right)=r e^{i f(\theta)} .
$$

Since the dilatation $K_{\psi}(z)$ of $\psi$ at $z=r e^{i \theta}$ satisfies

$$
K_{\psi}(z)=\frac{\left|1+f^{\prime}(\theta)\right|+\left|1-f^{\prime}(\theta)\right|}{\left|1+f^{\prime}(\theta)\right|-\left|1-f^{\prime}(\theta)\right|} \leqslant C,
$$

$\psi$ is a quasiconformal mapping from $\Omega_{0}$ to $\psi\left(\Omega_{0}\right)$ (cf., e.g., Lehto and Virtanen [4]).

Observe that the mapping $\psi$ fixes sequences $\left\{t_{n}\right\}_{1}^{\infty}$ and $\left\{t_{n}^{\prime}\right\}_{1}^{\infty}$ obtained in the Lemma in $\S 1$ termwise. Since the region $\psi\left(\Omega_{0}\right)$ contains the disk $B(1,1)$ and the interval $(0,1]$ contains both the sequences $\left\{\psi\left(t_{n}\right)\right\}_{1}^{\infty}$ and $\left\{\psi\left(t_{n}^{\prime}\right)\right\}_{1}^{\infty}$, the Lemma in $\S 2$ assures that $\left\{\psi\left(t_{n}\right)\right\}$ and $\left\{\psi\left(t_{n}^{\prime}\right)\right\}$ converge to an identical minimal point in the Martin compactification of $\psi\left(\Omega_{0}\right)$. On the other hand, by the Lemma in $\S 1$, limits of $\left\{t_{n}\right\}$ and $\left\{t_{n}^{\prime}\right\}$ are distinct. Therefore $\psi$ cannot be extended to a homeomorphism between Martin compactifications of $\Omega_{0}$ and $\psi\left(\Omega_{0}\right)$.

\section{REFERENCES}

1. A. Ancona, Principe de Harnack a la frontière et théorème de Fatou pour opérateur elliptique dans un domaine lipschitzien, Ann. Inst. Fourier (Grenoble) 28 (1978), 169-213.

2. Une propriété de la compactification de Martin d'un domaine euclidien, Ann. Inst. Fourier (Grenoble) 29 (1979), 71-90. 
3. C. Constantinescu and A. Cornea, Ideale Ränder Riemannscher Flächen, Springer-Verlag, Berlin and New York, 1963.

4. O. Lehto and K. I. Virtanen, Quasikonforme Abbildungen, Springer-Verlag, Berlin and New York, 1965.

5. H. L. Royden, Open Riemann surfaces, Ann. Acad. Sci. Fenn. Ser. A I No. 249 (1958), 13 pp.

6. L. Sario and M. Nakai, Classification theory of Riemann surfaces, Springer-Verlag, Berlin and New York, 1970.

Department of Mathematics, Daido Institute of Technology, Daido, Minami, Nagoya 457 , JAPAN 